\title{
Explaining Physics to a Lay Audience
}

\author{
Tiziano Virgili ${ }^{1}$ \\ University of Salerno and INFN \\ Via Giovanni Paolo II, 132 - 84084 - Fisciano (SA) - Italy \\ E-mail: tiziano.virgiliesa.infn.it
}

In this paper, a short discussion on how to explain particle physics to a general public will be presented. The communication strategy depends strongly on the specific audience, and here only selected examples will be reported. The important role of the web will be shortly discussed, as well as the effectiveness of items like books and games.

Sixth Annual Conference on Large Hadron Collider Physics (LHCP2018)

4-9 June 2018

Bologna, Italy

\section{${ }^{1}$ Tiziano Virgili}


1. Introduction

Explaining physics is in general a very difficult task. Even the most simple physics law requires some basic mathematics, which are clearly not appreciated by the general public. In the case of particle physics this problem is enormously increased, as the physics itself is neither simple, nor intuitive. The results are usually presented in a very complex way, hardly understandable by common people. We can therefore ask ourselves why we have to do such a big effort of explaining the physics to a "lay audience". There are indeed many good reasons for doing that:

- scientific discoveries are part of the human heritage, so it is our duty to divulgate them as much as possible;

- CERN and in general science and the scientific method should be a model for people, particularly young people (but not only them);

- research needs financial support, therefore we have to convince politicians to provide funding, which means in the end to convince people that research is important for society.

We can conclude that the effort of involving more and more "common people" in the subject of our researches cannot be anymore considered just an academic exercise, it is indeed a basic activity that should complete and integrate, as far as possible, our scientific work.

\section{Communication strategies}

It should be clear from the beginning, that any attempt of explaining the LHC physics cannot work until a sort of simplification - schematization is performed. The message that must be delivered in the first place is very simple, and it is why we do our work. This means to explain why our research is important, for science and in general for the world (because it leads to a better understanding of the Universe, because it allows many practical and common applications, and so on). Then, it is necessary to explain some basic concepts in a simplified way (for instance, what is the meaning of the Higgs Boson). Finally, if possible, more details can be added, such as a look at the experimental results, the description of detectors, etc. The communication strategies and the details of the message depend clearly on the audience to whom they are addressed. The general public ("lay audience") is not a homogeneous entity: people have different interests, knowledge, and so on, and the communication should be adapted accordingly. This means that it is fundamental to divide the audience into common groups, by age, by scientific background, by interest, and then select and design platforms accordingly. Figure 1 shows a schematic summary of the strategies that have been developed and applied in outreach activities. Some of them are organized at CERN, however there is an increasing number of external (local) activities, and there is also an increasing number of on-line strategies[1]. A full review of everything would be beyond the scope of this short article, so in the next chapters only few selected examples will be discussed. Here I want to mention the relevance of the web, and in particular the role of networks IPPOG[2] and QuarkNet[3], which are collaborations that give help for the divulgation of particle physics, by providing material, help on-line, by organizing masterclasses and so on. 
THE STRATEGIES

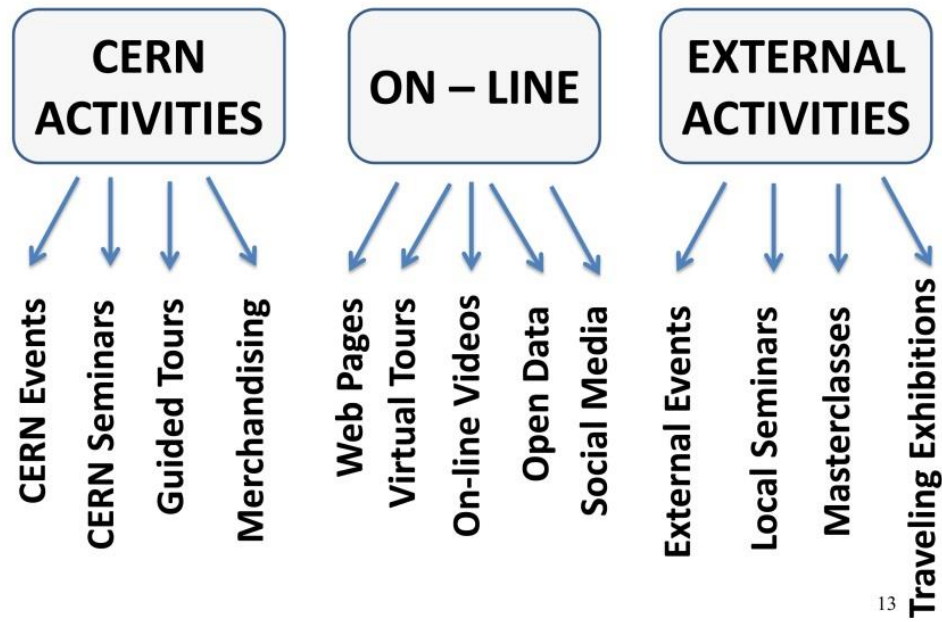

Figure 1: a summary of the main outreach activity strategies.

\section{Presenting Unintuitive Physics by Elementary Examples}

One of the basic and simplest ways of communication is based on images. One of the most well-known and effective examples comes from General Relativity: in the classic picture of masses on a thick mattress, it is easy to understand how a mass produces a distortion of the space time (which is described as the mattress), and how this distortion produces gravitational interaction. The heavier the mass, the more important the distortion and so the interaction. A similar picture can be used to describe the effect of the Higgs field (figure 2). This can be visualized as a sort of "dense fog", on which some particles can be embroiled. Photons and massless particles don't interact, and so they can "go straight" at the maximum speed, which is the speed of light. The particles that interact most are also those with lower speed, and this can be seen as the fact that they got a mass: the stronger the interaction, the higher the mass. By using images, it is also possible to explain at least the meaning of spontaneous symmetry breaking: the "sombrero hat", or the "round table" examples turn to be very effective. However, is not so simple to explain the connection with the Higgs field. Another simple example is the Quark-Gluon Plasma (QGP) production in nucleus-nucleus collisions. It is quite straightforward to introduce quarks, going from matter to atoms to nucleons. The less intuitive part is the problem of confinement. Probably the best example is the spring: you can imagine a meson as a quark-antiquark pair held together by a spring. If the spring is pulled, additional energy is introduced in the system, and at some point a new pair will be created, so in the end a new second meson is produced. It should be remarked that the quark confinement is an experimental fact, and up to now no single quarks have been observed. It is quite simple to illustrate QGP formation from nuclear collision. As the density is high enough, nucleons are broken and quarks are free in this "soup" of quarks and gluons (many simulations can be found on the web to better show the process). 
One more example is the $\mathrm{CP}$ violation, which can be presented by introducing as a first Parity symmetry, then Charge Conjugation and their combination.

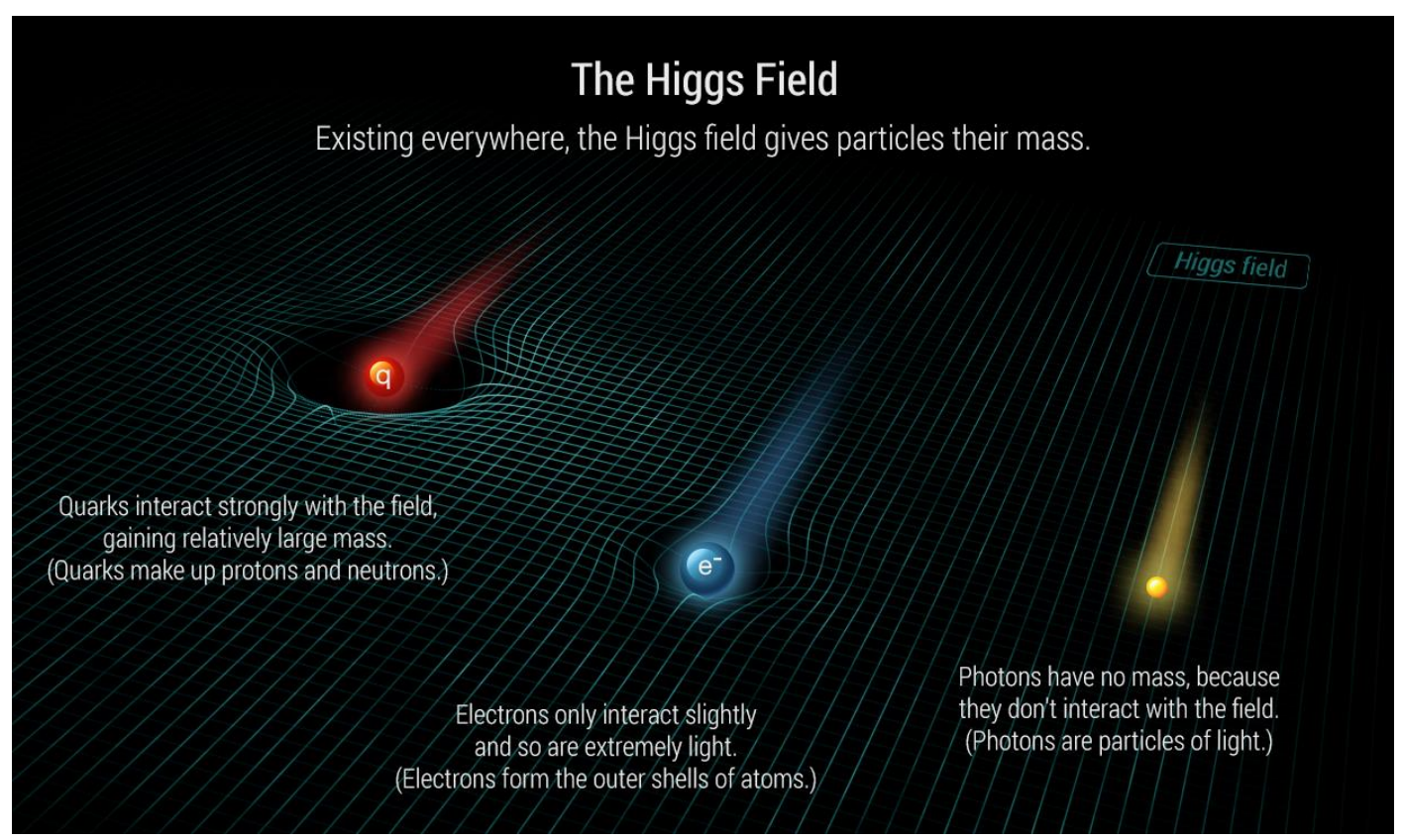

Figure 2: a pictorial image of the Higgs field and its interactions.

\section{Explaining Physics by Games}

A totally different way of communication simply uses merchandising. Items like t-shirts, cups, etc., are very attractive to the general public, and among them of course books and games are the most effective to explain physics. Many books and games have been developed at CERN by the main LHC collaborations, from classic card games to traditional society games, and from original games to paper models, pop-up books and more (figure 3). The main obstacle to a full, worldwide diffusion is clearly the availability of those items, which is normally limited to CERN. The production of games (and in general items) by well-known editors or brands can be very effective in the divulgation of physics, however to have the approval of international brands is not an easy goal. A different approach could be to produce locally some useful tools, such as dice, cards, paper models and so on, by using common printers. Some examples of templates to be printed, cut and assembled can be found on the web[4].

Another effective example of communication comes from Japan: Tantei Galileo (which means Detective Galileo) is a TV drama, where the main character is a physicist (his name is of course Yukawa!), who helps police to solve "impossible" cases, where apparently paranormal activities are involved. In each episode the public can learn some basic physics concepts. This project is very expensive, but also very effective in interesting people in physics. In this case also, it is possible to find on the web both video and English subtitles. 


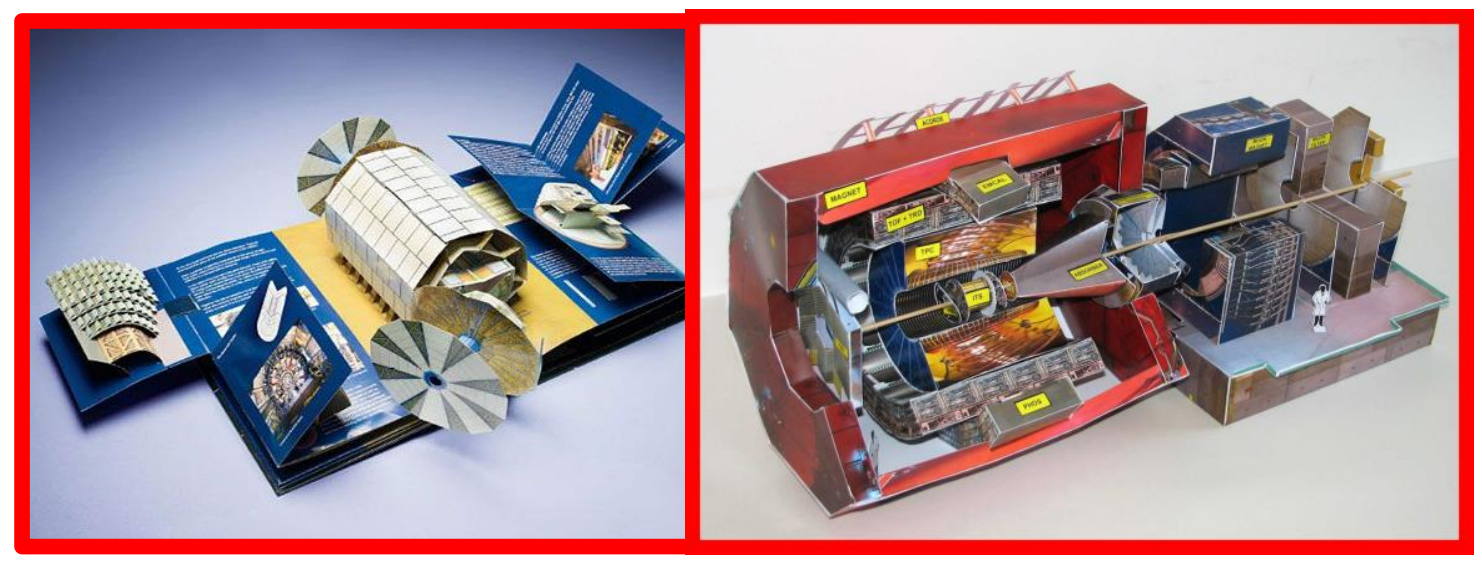

Figure 3: ATLAS Pop-up Book (left), and ALICE Paper Model (right).

\section{Conclusions}

Explaining physics, and specifically LHC physics, is a difficult task, that cannot be underestimated or ignored. Several tools are available on the web, to help teachers or common people to understand and to divulgate simple concepts.

\section{Acknowledgements}

The author wishes to thank the ALICE, ATLAS, CMS and LHCb collaborations for their help and suggestions, with special mention to Angelos Alexopoulos, Katarina Anthony, Claire Adam Bourdarios, Steven Goldfarb, Despina Hatzifotiadou, Dirk Hoffmann, Marzena Lapka, Sascha Mehlhase and Thomas Naumann.

\section{References}

[1] https://communications.web.cern.ch/

[2] http://ippog.org/

[3] https://quarknet.org/content/home-page

[4] See for instance https://virgili.web.cern.ch/ 